\title{
Minimal degree sequence for 2-bridge knots
}

\author{
by \\ Prabhakar Madeti and Rama Mishra (New Delhi)
}

\begin{abstract}
We discuss polynomial representations for 2-bridge knots and determine the minimal degree sequence for all such knots. We apply the connection between rational tangles and 2-bridge knots.
\end{abstract}

1. Introduction. It is known that, up to ambient isotopy, every noncompact knot is equivalent to some polynomial knot [10]. In fact, one can say that the set of all non-compact knot types is the same as the set of all polynomial knot types because the isotopy between two equivalent polynomial knots can be given by a one-parameter family of polynomial embeddings. Thus, for a given knot type $K$ (always non-compact for us) there exist three real polynomials $f(t), g(t)$ and $h(t)$ such that the map $t \mapsto(f(t), g(t), h(t))$ represents $K$. If $\operatorname{deg} f(t)=l, \operatorname{deg} g(t)=m$ and $\operatorname{deg} h(t)=n$, then we say that the triple $(l, m, n)$ is a degree sequence of $K$. We define $(l, m, n)$ to be the minimal degree sequence for the knot type $K$ if it is minimal amongst all degree sequences of $K$ with respect to the lexicographic ordering in $\mathbb{N}^{3}$. A knot can be well understood if its minimal degree sequence is known. In fact a polynomial knot with the minimal degree sequence has a diagram that cannot be reduced further. Thus, to determine the minimal degree sequence of a given knot type is an interesting problem.

In our earlier papers we have found a degree sequence for all torus knots $([6],[7])$ and also the minimal degree sequence for torus knots of type $(2,2 n+1)([7])$. The minimal degree sequence for a general torus knot of type $(p, q)$ is still not known, except for the special case when $q=2 p-1$ $([5])$. For convenience we state some known partial results:

Theorem ([7]). The minimal degree sequence for a torus knot of type $(2,2 n+1)$ is either $(3,2 n+2,2 n+4)$ or $(3,2 n+2,2 n+3)$ or $(3,2 n+3,2 n+4)$ according as $n$ is of the form $3 m, 3 m+1$ or $3 m+2$ respectively.

Key words and phrases: 2-bridge knots, rational tangles, polynomial knots. 
Theorem ([5]). The minimal degree sequence for a torus knot of type $(p, 2 p-1), p \geq 2$, is given by $(2 p-1,2 p, d)$, where $2 p+1 \leq d \leq 4 p-3$.

When we determine the minimal degree sequence for torus knots of type $(2,2 n+1)$, we use the fact that they have bridge number 2 and they are alternating. Since all 2-bridge knots are alternating we have tried imitating a similar technique and obtained the minimal degree sequence for all 2-bridge knots. In this paper we prove the following:

TheOREM. The minimal degree sequence for a 2-bridge knot $K$ with $N$ crossings is either $(3, N+1, N+2)$ or $(3, N+1, N+3)$ or $(3, N+2, N+3)$ according as $N \equiv 0$, or 1 or $2(\bmod 3)$ respectively.

\section{Background material}

Definition 2.1. Let $D_{K}$ be a knot diagram for a given knot $K$. An overpass in $D_{K}$ is a subarc of the diagram which contains an over crossing but no under crossing points. The number of overpasses in a given knot diagram is called the bridge number of the diagram.

Definition 2.2. The bridge number $\operatorname{br}(K)$ of a knot $K$ is the least bridge number of all the knot diagrams of $K$. In fact $\operatorname{br}(K)$ turns out to be the minimum value of the number of local maxima over all the regular diagrams of $K$.

\section{REMARK 2.3.}

1. By convention the bridge number of the unknot is 1 .

2 . $\operatorname{br}(K)$ is a knot invariant.

The bridge number for the knot diagram shown in Figure 1(i) is 3 whereas for Figure 1(ii) it is 2. Both figures are the knot diagrams of the trefoil knot. Thus the bridge number for the trefoil is 2 .

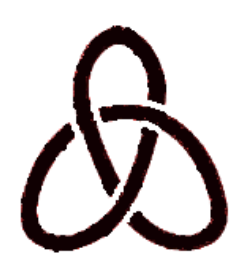

(i)

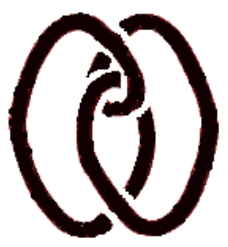

(ii)

Fig. 1

Definition 2.4. A knot $K$ is called a 2-bridge knot if the bridge number of $K$ is 2 . 
2-bridge knots are also known as rational knots which arise from rational tangles. Rational tangles were introduced by John B. Conway ([2]-[4] and [9]). To study them, first we need to define a tangle.

Definition 2.5. A tangle is analogous to a link except that it has free ends. These ends are called strands that enter a box (the tangle box) within which there are no free ends. Inside the box one may find closed loops that are knotted and linked with the tangle strands. The strands of the tangle may themselves be knotted and linked.

Definition 2.6. Two tangles $T_{1}$ and $T_{2}$ are said to be ambient isotopic if it is possible to deform $T_{1}$ into $T_{2}$ without moving the end points and without moving the strands outside the tangle box.

We are generally concerned with rational tangles, i.e., tangles with four ends. Such tangles have 2-strands. To define a rational tangle formally, we need a few definitions.

Definition 2.7. A tangle with two unlinked horizontal (resp. vertical) strands is called a [0] tangle (resp. [ $\infty$ ] tangle). These two tangles are also known as trivial tangles. They are shown in Figure 2:

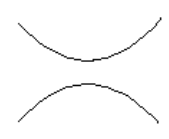

[0]

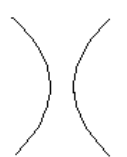

$[\infty]$

Fig. 2

DeFinition 2.8. Given an integer $n$, a horizontal (resp. vertical) integer tangle $t_{n}$ (resp. $t_{n}^{\prime}$ ) is a twist of two horizontal (resp. vertical) strands $|n|$ times in the positive or negative direction according to the sign of $n$. The directions are shown in Figure 3 (resp. in Figure 4).

Definition 2.9. The horizontal sum " + " and the vertical sum " $\oplus$ " of two tangles $T_{1}$ and $T_{2}$ are defined as in Figure 5. Thus, twisting two adjacent strands of a tangle $T_{1}$ is equivalent to adding (with + ) an integer tangle $t_{n_{2}}$ on the right or the left of $t_{n_{1}}$ or adding (with $\oplus$ ) $t_{n_{2}}^{\prime}$ on the top or bottom of $t_{n_{1}}^{\prime}$, where $n_{1}$ and $n_{2}$ are any integers.

REMARK 2.10.

1. It is easy to see that $t_{n_{1}}+t_{n_{2}} \stackrel{\text { isotopic }}{\sim} t_{n_{1}+n_{2}}$ and $t_{n_{1}}^{\prime} \oplus t_{n_{2}}^{\prime} \stackrel{\text { isotopic }}{\sim} t_{n_{1}+n_{2}}^{\prime}$.

2. $t_{0}=[0]$ and $t_{0}^{\prime}=[\infty]$.

3. A negative twist cancels a positive twist topologically. Thus cancellation of positive and negative integers is paralleled in tangle topology. 

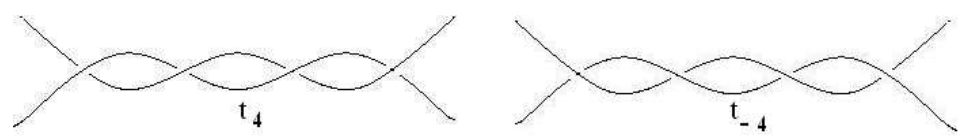

Fig. 3
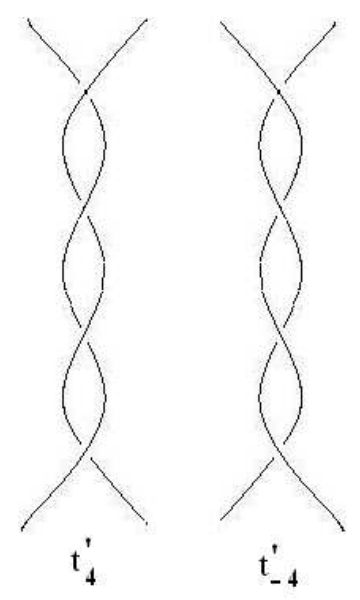

Fig. 4

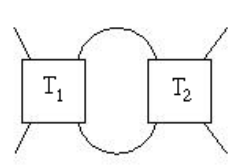

$$
\mathrm{T}_{1}+\mathrm{T}_{2}
$$

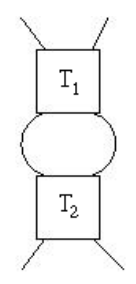

$$
\mathrm{T}_{1} \oplus \mathrm{T}_{2}
$$

Fig. 5

This particular cancellation is an instance of the Reidemeister move in knot theory.

DEFINITION 2.11. A rational tangle is a proper embedding of two unoriented $\operatorname{arcs} \alpha_{1}, \alpha_{2}$ in a 3 -ball $\mathbb{B}^{3}$, so that the four end points lie on the boundary of $\mathbb{B}^{3}$, and such that there exists a homeomorphism of pairs

$$
f:\left(\mathbb{B}^{3}, \alpha_{1}, \alpha_{2}\right) \rightarrow\left(D^{2} \times I,\{x, y\} \times I\right),
$$

where $I$ is any closed interval.

Definition 2.12. Two rational tangles $T_{1}$ and $T_{2}$ in $\mathbb{B}^{3}$ are said to be isotopic (denoted as $T_{1} \sim T_{2}$ ) if there exists an orientation-preserving 
self-homeomorphism $h:\left(\mathbb{B}^{3}, T_{1}\right) \rightarrow\left(\mathbb{B}^{3}, T_{2}\right)$ such that $h$ is the identity map on the boundary.

Definition 2.13. The numerator closure of a rational tangle $T$, denoted by $N(T)$, is obtained by joining the two upper endpoints and the two lower endpoints of $T$ with simple arcs, as shown in Figure 6.
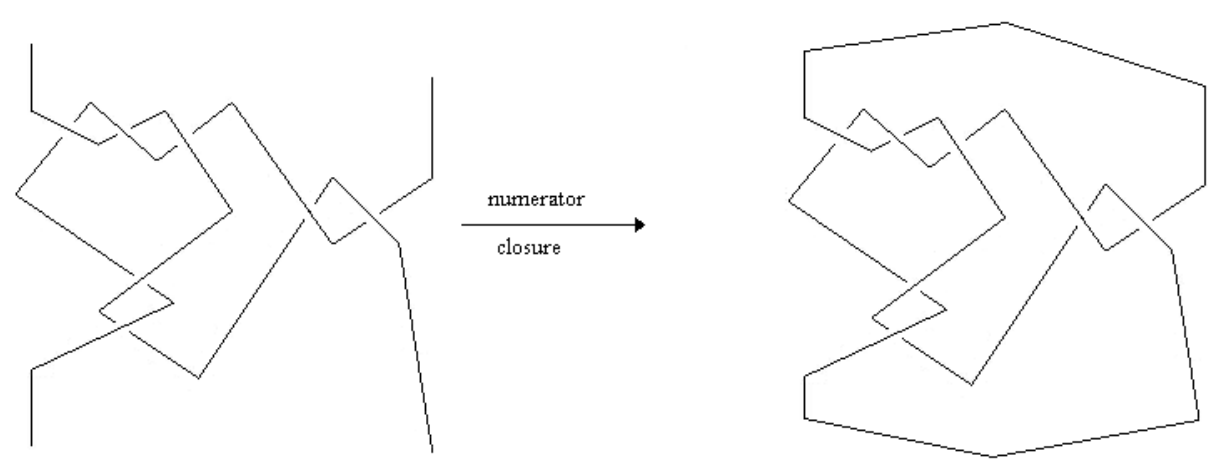

Fig. 6

Definition 2.14. The denominator closure of a rational tangle $T$, denoted by $D(T)$, is obtained by joining each pair of the corresponding top and bottom endpoints of $T$ with simple arcs, as shown in Figure 7 .

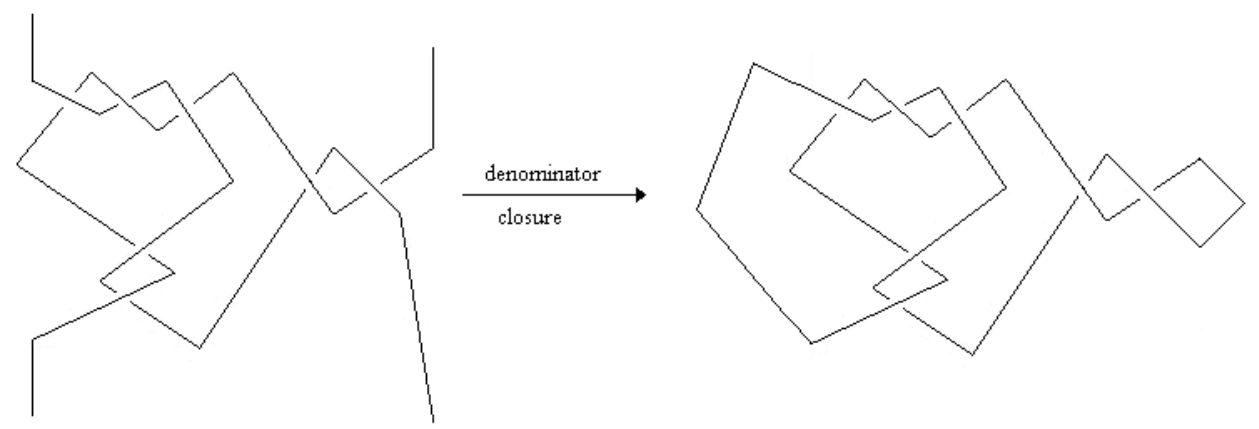

Fig. 7

Definition 2.15. A rational tangle is said to be in standard form if it is created by a consecutive + of the tangles $\pm t_{1}$ only on the right (or only on the left) and $\oplus$ by the tangles $\pm t_{1}$ only at the bottom (or only at the top), starting from the tangle $t_{0}$ or $\left[t_{0}^{\prime}\right]$.

We can check the following: 
Proposition 2.16. A rational tangle can be obtained by performing either of the following:

1. A finite alternating sequence of horizontal and vertical twists on [0].

2. A finite alternating sequence of vertical and horizontal twists on $[\infty]$.

REMARK 2.17.

1. By Proposition 2.16, every rational tangle can be completely determined by how we perform a sequence, say $\left[t_{n_{1}}, \ldots, t_{n_{k}}\right]$, of vertical and horizontal twists in an alternate manner.

2. If $k$ is odd, we can obtain the relevant rational tangle by first performing $t_{n_{1}}$ horizontal twists on [0], then $t_{n_{2}}$ vertical twists, $t_{n_{3}}$ horizontal twists, and repeating the twists alternately until finally we perform a horizontal twist $t_{n_{k}}$ times. In this case we call this rational tangle a horizontal rational tangle.

3 . If $k$ is even, we can obtain the relevant rational tangle by first performing $t_{n_{1}}$ vertical twists on [ $\infty$ ], then $t_{n_{2}}$ horizontal twists, and repeating the twists alternately until finally we perform a horizontal twist $t_{n_{k}}$ times. We call such a rational tangle a vertical rational tangle.

Definition 2.18. A tangle is said to be alternating if the crossings in the tangle alternate from under to over as we go along any component or arc of the tangle.

There is a simple way of representing rational tangles by using a sequence of integer tangles.

Definition 2.19. For any sequence of integers $n_{1}, \ldots, n_{k}$, let $T_{n_{1}}, \ldots$, $T_{n_{k}}$ be a sequence of integer tangles, where $T_{n_{i}}=t_{n_{i}}$ or $t_{n_{i}}^{\prime}$. Consider the sequence of rational tangles given by:

$$
\begin{aligned}
\tau_{n_{1}} & =T_{n_{1}}, \\
\tau_{n_{j+1}} & = \begin{cases}\tau_{n_{j}}+T_{n_{j+1}} & \text { if } T_{n_{j+1}}=t_{n_{j+1}}, \\
\tau_{n_{j}} \oplus T_{n_{j+1}} & \text { if } T_{n_{j+1}}=t_{n_{j+1}}^{\prime},\end{cases}
\end{aligned}
$$

for $j \geq 1$.

The rational tangle $\tau_{n_{k}}$ is called a rational tangle with $k$ integer tangles $T_{n_{1}}, \ldots, T_{n_{k}}$ and is denoted by $\left[T_{n_{1}}, \ldots, T_{n_{k}}\right]$.

REMARK 2.20.

1. The number of integer tangles in a rational tangle is not unique.

2. A rational tangle with $k$ integer tangles is the same as a rational tangle that is obtained by performing a finite alternating sequence of vertical and horizontal twists on [0] or [ $\infty]$. Thus any rational tangle is a rational tangle with $k$ integer tangles for some $k$. 
It is easy to represent a rational tangle via a continued fraction built from the integer tangles. Before defining a continued fraction in integer tangles, let us formally define the following three operations.

Definition 2.21. If $T$ is a rational tangle, then:

(i) $-T=T^{*}$, the mirror image of $T$.

(ii) The clockwise inverse of $T$, denoted by $1 / T^{\mathrm{c}}$, is the tangle obtained from $T$ by rotating $90^{\circ}$ clockwise and taking the mirror image.

(iii) The counterclockwise inverse of $T$, denoted by $1 / T^{\mathrm{cc}}$, is the tangle obtained from $T$ by rotating $90^{\circ}$ counterclockwise and taking the mirror image.

THEOREM 2.22. If $T$ is a rational tangle, then $1 / T^{\mathrm{c}}$ and $1 / T^{\mathrm{cc}}$ are ambient isotopic.

Note. Since $1 / T^{\mathrm{c}}$ and $1 / T^{\mathrm{cc}}$ are ambient isotopic, we will represent both by $1 / T$.

It is easy to check the following:

1. $T$ is a horizontal rational tangle $\Leftrightarrow 1 / T$ is a vertical rational tangle.

2. $t_{n_{k}}^{\prime}=1 / t_{n_{k}}$ and $t_{n_{k}}=1 / t_{n_{k}}^{\prime}$.

3. $t_{n_{i}}+t_{n_{j}}^{\prime}=t_{n_{i}}+1 / t_{n_{j}}$.

4. $t_{n_{i}} \oplus t_{n_{j}}^{\prime}=\frac{1}{t_{n_{j}}+1 / t_{n_{i}}}$.

5. $-\left(T_{1}+T_{2}\right)=\left(-T_{1}\right)+\left(-T_{2}\right),-\left(1 / T_{1}\right)=1 /\left(-T_{1}\right),-t_{n_{i}}=t_{-n_{i}}$, and $-t_{n_{i}}^{\prime}=t_{-n_{i}}^{\prime}$.

Now we can build our rational tangles using the tangle operations,$+ \oplus$, - , and / as follows:

Any horizontal rational tangle $T$ can be written in the form

$$
T=t_{n_{k}}+\frac{1}{t_{n_{k-1}}+\frac{1}{\cdots+\frac{1}{t_{n_{1}}}}}
$$

and any vertical rational tangle $T^{\prime}$ can be written in the form

$$
T^{\prime}=\frac{1}{t_{n_{k}}+\frac{1}{t_{n_{k-1}}+\frac{1}{\cdots+\frac{1}{t_{n_{1}}}}}} .
$$

Hence every rational tangle can be described via a continued fraction in integer tangles and such rational tangles are said to be in continued fraction form.

REMARK 2.23.

1. Every rational tangle can be written in continued fraction form. 
2. As the continued fraction form and the standard form of a rational tangle are equivalent, the above correspondence shows that it is straightforward to write down the one from the other.

Definition 2.24. A rational tangle $T=\left[t_{n_{1}}, \ldots, t_{n_{k}}\right]$ is in canonical form if $T$ is alternating and $k$ is odd. Moreover, $T$ is called positive or negative according to the sign of its terms.

We note that if $T$ is alternating and $k$ is even, then we can bring $T$ to canonical form by breaking $t_{n_{k}}$ to $\operatorname{sign}\left(t_{n_{k}}\right) \cdot\left(\left|t_{n_{k}}\right|-1\right)+\operatorname{sign}\left(t_{n_{k}}\right) \cdot 1$, and thus, $\left[t_{n_{1}}, \ldots, t_{n_{k}}\right]$ to $\left[t_{n_{1}}, \ldots, \operatorname{sign}\left(t_{n_{k}}\right) \cdot\left(\left|t_{n_{k}}\right|-1\right), \operatorname{sign}\left(t_{n_{k}}\right) \cdot 1\right]$. As a consequence, we have the following :

1. Every rational tangle can be isotoped to canonical form.

2. Every rational tangle can be brought via isotopy to standard form.

The above forms of rational tangles suggest associating to the rational tangles (I) and (II) the arithmetic continued fractions

$$
n_{k}+\frac{1}{n_{k-1}+\frac{1}{\ldots+\frac{1}{n_{1}}}} \text { and } \frac{1}{n_{k}+\frac{1}{n_{k-1}+\frac{1}{\ldots+\frac{1}{n_{1}}}}} .
$$

We will represent these continued fractions by $\left[n_{1}, \ldots, n_{k}\right]$.

REMARK 2.25. If a continued fraction $\left[n_{1}, \ldots, n_{k}\right]$ has even length, then we can bring it to odd length via the last term transformations:

$$
\begin{array}{ll}
{\left[n_{1}, n_{2}, \ldots, n_{k}\right]=\left[+1, n_{1}-1, n_{2}, \ldots, n_{k}\right]} & \text { for } n_{1}>0, \\
{\left[n_{1}, n_{2}, \ldots, n_{k}\right]=\left[-1, n_{1}+1, n_{2}, \ldots, n_{k}\right]} & \text { for } n_{1}<0 .
\end{array}
$$

We say that a continued fraction is termwise positive (resp. negative) if all the numerical terms in its expression are positive (resp. negative).

Definition 2.26. A continued fraction $\left[n_{1}, \ldots, n_{k}\right]$ is said to be in canonical form if it is termwise positive or negative and $k$ is odd.

We can observe that each of these continued fractions is equal to a rational fraction which we call the fraction of the tangle and denote by $F(t)$. In particular, $F\left(t_{n}\right)=n$ and $F\left(t_{n}^{\prime}\right)=F\left(1 / t_{n}\right)=1 / n$.

For any fraction of the tangle $F(t)=\alpha / \beta$, there exist $n_{k} \in \mathbb{Z}, n_{1}, \ldots$, $n_{k-1} \in \mathbb{Z}-\{0\}$ such that $\alpha / \beta=\left[n_{1}, \ldots, n_{k}\right]$; moreover, we have the following:

1. $\frac{\alpha}{\beta} \pm 1=\left[n_{1}, n_{2}, \ldots, n_{k} \pm 1\right]$,

2. $\frac{\beta}{\alpha}=\left[n_{1}, n_{2}, \ldots, n_{k}, 0\right]$,

3. $\frac{-\alpha}{\beta}=\left[-n_{1},-n_{2}, \ldots,-n_{k}\right]$. 
Thus we can show that: Every continued fraction $\left[n_{1}, \ldots, n_{k}\right]$ can be transformed to a unique canonical form $\left[m_{1}, \ldots, m_{l}\right]$, where $m_{i}$ 's are either all positive or all negative integers and $l$ is odd.

Definition 2.27. A knot obtained by taking the numerator or denominator closure of a rational tangle is called a rational knot.

REMARK 2.28.

1. It is easy to observe that rational knots and 2-bridge knots are equivalent.

2. Since every 2-bridge knot is obtained as the numerator closure of a rational tangle, we can represent a 2-bridge knot by a standard regular diagram of type $\left[n_{1}, \ldots, n_{k}\right]$ of the form shown in Figure 8 , and denote it by $C\left(n_{1}, \ldots, n_{k}\right)$.

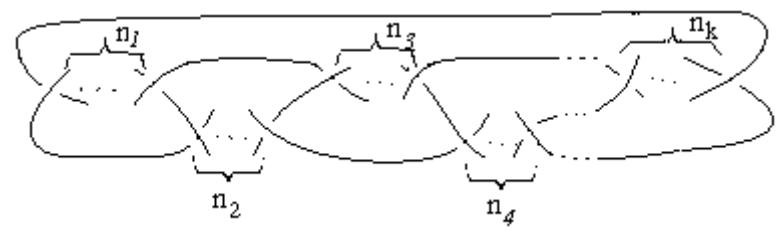

Fig. 8

Thus a 2-bridge knot is denoted by $C\left(n_{1}, \ldots, n_{k}\right)$ where $k$ is odd. This is known as Conway's normal form. Thus with a 2-bridge knot $C\left(n_{1}, \ldots, n_{k}\right)$ where $k$ is odd, we can associate a rational number

$$
\frac{\alpha}{\beta}=\left[n_{1}, n_{2}, \ldots, n_{l}\right], \quad \operatorname{gcd}(\alpha, \beta)=1 .
$$

Conversely, from a rational number $\alpha / \beta$ (with $|\alpha / \beta|>1$ ), we can create a 2-bridge knot by taking its continued fraction expansion. In fact, we have obtained a correspondence between the 2-bridge knot $C\left(n_{1}, \ldots, n_{l}\right)$ and the rational number $\alpha / \beta$. In the continued fraction expansion of $\alpha / \beta$, we may always assume that $l$ is odd.

Theorem 2.29 (Schubert, 1956, see [1]). Suppose that $K$ and $K^{\prime}$ are 2 -bridge knots of type $(\alpha, \beta)$ and $\left(\alpha^{\prime}, \beta^{\prime}\right)$ respectively, which are obtained by taking the numerator of a rational tangle. Then $K$ and $K^{\prime}$ are equivalent if and only if either

1. $\alpha=\alpha^{\prime}, \beta \equiv \beta^{\prime}(\bmod \alpha)$ or

2. $\alpha=\alpha^{\prime}, \beta \beta^{\prime} \equiv 1(\bmod \alpha)$.

REMARK 2.30. If $\alpha$ is odd then $K$ is a knot, otherwise it is a link. Since our focus is on knots, we assume that $\alpha$ is odd. The observation made in 
Remark 2.28 is the main idea that we shall be using in the proof of our main result.

\section{Main result}

Theorem 3.1. Let $K$ be a 2-bridge knot with $N$ crossings. Then the minimal degree sequence for $K$ is given by

(i) $(3, N+1, N+2)$ when $N \equiv 0(\bmod 3)$;

(ii) $(3, N+1, N+3)$ when $N \equiv 1(\bmod 3)$;

(iii) $(3, N+2, N+3)$ when $N \equiv 2(\bmod 3)$.

Proof. Choose a regular diagram of $K$ using its representation as a rational knot in Conway's normal form $C\left(a_{1}, \ldots, a_{n}\right)$, where $n$ is odd, as shown in Figure 9. The non-compact version is shown in Figure 10. Let $p / q$ be the rational number associated to this canonical form, i.e.,

$$
\frac{p}{q}=\left[a_{1}, \ldots, a_{n}\right],
$$

where $p$ and $q$ are relatively prime. Since $K$ is alternating, all $a_{i}$ 's have the same sign. Without any loss of generality we can assume that they are all positive. Since the number of crossings is $N$, we have $\sum_{i=1}^{n} a_{i}=N$.

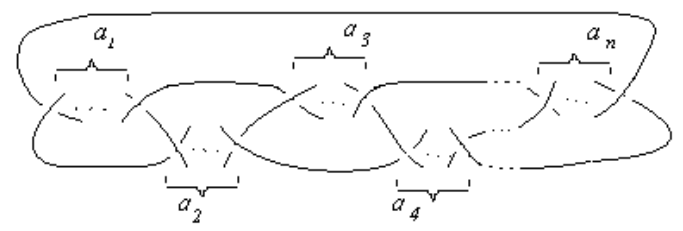

Fig. 9

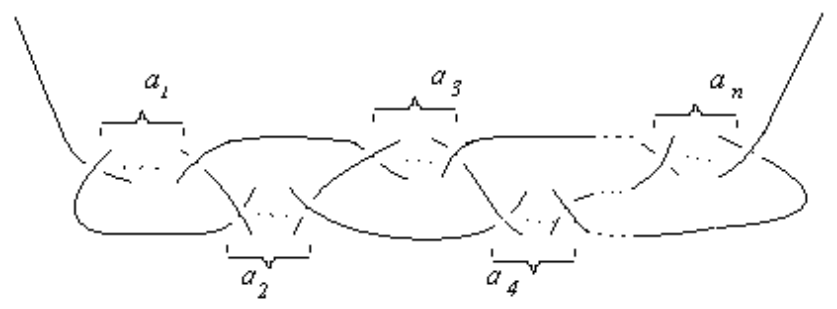

Fig. 10

We consider a regular projection of $K$ into the $X Y$ plane as shown in Figure 11. First we want to represent this plane curve by polynomial 


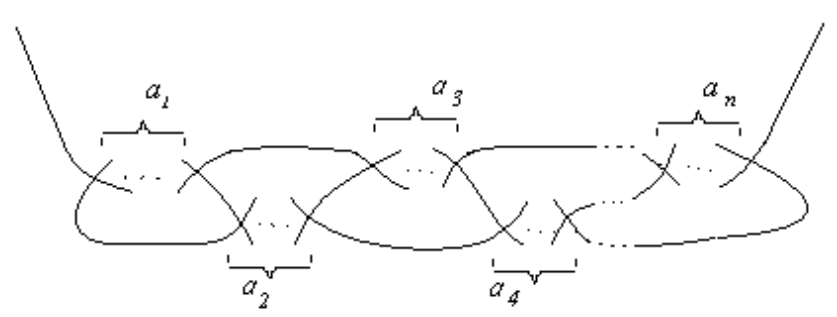

Fig. 11

parametrization. We observe that in this plane curve there is only one local maximum and one local minimum in the $X$-direction. Thus we can represent the $X$-coordinate by a polynomial $f(t)$ of degree 3 . For the sake of symmetry, take $f(t)=t\left(t^{2}-\alpha^{2}\right)$, where $\alpha$ is a real number. Thus, the $X$-coordinate vanishes at $t=-\alpha, 0$ and $+\alpha$. Choose a real number $\beta>\alpha$ such that for any $t_{0}$ outside $[-\beta, \beta]$, the equation $f(t)=f\left(t_{0}\right)$ has only one real root, viz. $t=t_{0}$. This ensures that the plane curve having $f(t)$ as $X$-coordinate has no self-intersection outside the interval $[-\beta, \beta]$. Now divide the interval $[-\beta, \beta]$ in $2 n$ parts, $\left[-\beta, \alpha_{1}\right],\left[\alpha_{1}, \alpha_{2}\right], \ldots,\left[\alpha_{2 n-1}, \beta\right]$, in such a way that for each $i=1, \ldots, n$, there are exactly two intervals, say $\left(\alpha_{m_{i}-1}, \alpha_{m_{i}}\right)$ and $\left(\alpha_{n_{i}-1}, \alpha_{n_{i}}\right)$, where $a_{i}$ parameter values $s_{1}^{i}, \ldots, s_{a_{i}}^{i}$ from $\left(\alpha_{m_{i}-1}, \alpha_{m_{i}}\right)$ and $a_{i}$ parameter values $t_{1}^{i}, \ldots, t_{a_{i}}^{i}$ from $\left(\alpha_{n_{i}-1}, \alpha_{n_{i}}\right)$ satisfy

$$
f\left(s_{j}^{i}\right)=f\left(t_{j}^{i}\right) \quad \text { for all } j=1, \ldots, a_{i} .
$$

Looking at the pattern of the intersections in the curve we can decide where to choose the $s_{j}^{i}$ and $t_{j}^{i}$ from. It is easy to see that

$$
t_{j}^{i}=\frac{1}{2}\left(-s_{j}^{i} \pm \sqrt{\left(4 \alpha^{2}-3\left(s_{j}^{i}\right)^{3}\right)}\right) .
$$

For each $s_{j}^{i}$ we have two $t_{j}^{i}$, s. We will choose one $t_{j}^{i}$ corresponding to each $s_{j}^{i}$ which gives the double points in the curve. Therefore once we choose $s_{j}^{i}$ 's, the choice of $t_{j}^{i}$ 's is fixed (in fact, each $t_{j}^{i}$ is an analytic function of $s_{j}^{i}$ ). Now, to represent the $Y$-coordinate of this plane curve we choose a polynomial $g(t)$ of degree $N+1$ as

$$
g(t)=t^{N+1}+\beta_{1} t^{N}+\beta_{2} t^{N-1}+\cdots+\beta_{N} t+\beta_{N+1},
$$

where the coefficients $\beta_{1}, \ldots, \beta_{N}$ are such that

$$
g\left(s_{j}^{i}\right)=g\left(t_{j}^{i}\right) \quad \forall j=1, \ldots, a_{i} \text { and } i=1, \ldots, n .
$$

This gives a system of $N$ linear equations in $N$ variables $\beta_{1}, \ldots, \beta_{N}$ :

$$
\begin{array}{r}
\left(\left(s_{1}^{i}\right)^{N}-\left(t_{1}^{i}\right)^{N}\right) \beta_{1}+\left(\left(s_{1}^{i}\right)^{N-1}-\left(t_{1}^{i}\right)^{N-1}\right) \beta_{2}+\cdots+\left(\left(s_{1}^{i}\right)-\left(t_{1}^{i}\right)\right) \beta_{N} \\
=\left(t_{1}^{i}\right)^{N+1}-\left(s_{1}^{i}\right)^{N+1},
\end{array}
$$




$$
\begin{gathered}
\left(\left(s_{2}^{i}\right)^{N}-\left(t_{2}^{i}\right)^{N}\right) \beta_{1}+\left(\left(s_{2}^{i}\right)^{N-1}-\left(t_{2}^{i}\right)^{N-1}\right) \beta_{2}+\cdots+\left(\left(s_{2}^{i}\right)-\left(t_{2}^{i}\right)\right) \beta_{N} \\
\quad=\left(t_{2}^{i}\right)^{N+1}-\left(s_{2}^{i}\right)^{N+1}, \\
\vdots \\
\left(\left(s_{a_{i}}^{i}\right)^{N}-\left(t_{a_{i}}^{i}\right)^{N}\right) \beta_{1}+\left(\left(s_{a_{i}}^{i}\right)^{N-1}-\left(t_{a_{i}}^{i}\right)^{N-1}\right) \beta_{2}+\cdots+\left(\left(s_{a_{i}}^{i}\right)-\left(t_{a_{i}}^{i}\right)\right) \beta_{N} \\
=\left(t_{a_{i}}^{i}\right)^{N+1}-\left(s_{a_{i}}^{i}\right)^{N+1},
\end{gathered}
$$

for each $i=1, \ldots, n$. From the theory of linear equations, the above system has a unique solution if and only if the rank of the coefficient matrix is $N$, i.e. $\operatorname{det}(A) \neq 0$, where

$$
A=\left(\begin{array}{cccc}
\left(s_{1}^{1}\right)^{N}-\left(t_{1}^{1}\right)^{N} & \left(s_{1}^{1}\right)^{N-1}-\left(t_{1}^{1}\right)^{N-1} & \ldots & s_{1}^{1}-t_{1}^{1} \\
\left(s_{2}^{1}\right)^{N}-\left(t_{2}^{1}\right)^{N} & \left(s_{2}^{1}\right)^{N-1}-\left(t_{2}^{1}\right)^{N-1} & \ldots & s_{2}^{1}-t_{2}^{1} \\
\vdots & \vdots & \vdots & \vdots \\
\left(s_{a_{1}}^{1}\right)^{N}-\left(t_{a_{1}}^{1}\right)^{N} & \left(s_{a_{1}}^{1}\right)^{N-1}-\left(t_{a_{1}}^{1}\right)^{N-1} & \ldots & s_{a_{1}}^{1}-t_{a_{1}}^{1} \\
\left(s_{1}^{2}\right)^{N}-\left(t_{1}^{2}\right)^{N} & \left(s_{1}^{2}\right)^{N-1}-\left(t_{1}^{2}\right)^{N-1} & \ldots & s_{1}^{2}-t_{1}^{2} \\
\vdots & \vdots & \vdots & \vdots \\
\left(s_{a_{2}}^{2}\right)^{N}-\left(t_{a_{2}}^{2}\right)^{N} & \left(s_{a_{2}}^{2}\right)^{N-1}-\left(t_{a_{2}}^{2}\right)^{N-1} & \ldots & s_{a_{2}}^{2}-t_{a_{2}}^{2} \\
\vdots & \vdots & \vdots & \vdots \\
\left(s_{a_{n}}^{n}\right)^{N}-\left(t_{a_{n}}^{n}\right)^{N} & \left(s_{a_{n}}^{n}\right)^{N-1}-\left(t_{a_{n}}^{n}\right)^{N-1} & \ldots & s_{a_{n}}^{n}-t_{a_{n}}^{n}
\end{array}\right) .
$$

As each $t_{j}^{i}$ is a function of $s_{j}^{i}$, and in $\operatorname{det}(A)$ the coefficient of the monomial

$$
\begin{aligned}
\left(\left(s_{1}^{1}\right)^{N}\left(s_{2}^{1}\right)^{N-1} \cdots\left(s_{a_{1}}^{1}\right)^{N-\left(a_{1}-1\right)}\right)\left(\left(s_{1}^{2}\right)^{N-a_{1}}\right. & \left.\cdots\left(s_{a_{2}}^{2}\right)^{N-a_{1}-a_{2}+1}\right) \\
& \cdots\left(\left(s_{1}^{n}\right)^{N-a_{1}-\ldots-a_{n-1}-1} \cdots\left(s_{a_{n}}^{n}\right)\right)
\end{aligned}
$$

is non-zero, $\operatorname{det}(A)$ is a non-zero analytic function of $s_{j}^{i}$ where $j=1, \ldots, a_{i}$ and $i=1, \ldots, n$.

By a standard Zariski argument, the set of $N$ tuples of the form

$$
\left(s_{1}^{1}, \ldots, s_{a_{1}}^{1}, s_{1}^{2}, \ldots, s_{a_{2}}^{2}, \ldots, s_{1}^{n}, \ldots, s_{a_{n}}^{n}\right)
$$

for which $\operatorname{det}(A) \neq 0$ is an open and dense set in $\mathbb{R}^{N}$. We can observe that the above system has a unique solution for $\beta_{1}, \ldots, \beta_{N}$ by choosing $s_{j}^{i}$ from the desired open intervals such that $\operatorname{det}(A) \neq 0$. Thus we can get a polynomial $g(t)$ of degree $N+1$ such that $f\left(s_{j}^{i}\right)=f\left(t_{j}^{i}\right)$ and $g\left(s_{j}^{i}\right)=g\left(t_{j}^{i}\right)$. In fact, we can choose $s_{j}^{i}$ such that $(f(t), g(t))$ is a generic immersion. Here $(f(t), g(t))$ gives the desired projection, where

$$
\left(s_{1}^{1}, t_{1}^{1}\right),\left(s_{2}^{1}, t_{2}^{1}\right), \ldots,\left(s_{a_{1}}^{1}, t_{a_{1}}^{1}\right),\left(s_{1}^{2}, t_{1}^{2}\right), \ldots,\left(s_{a_{2}}^{2}, t_{a_{2}}^{2}\right), \ldots,\left(s_{a_{n}}^{N}, t_{a_{n}}^{N}\right)
$$

are the pairs of parameter values which give the intersections. 
To construct a polynomial $h(t)$ which provides an over/under crossing data for the corresponding 2-bridge knot, we choose

$$
h(t)=t^{N+2}+\gamma_{1} t^{N+1}+\gamma_{2} t^{N}+\cdots+\gamma_{N+1} t+\gamma_{N+2}
$$

such that $h\left(s_{i}^{j}\right)-h\left(t_{i}^{j}\right)<0$ for $i$ odd, and $h\left(s_{i}^{j}\right)-h\left(t_{i}^{j}\right)>0$ for $i$ even, where $j=1, \ldots, n$ and $i=1, \ldots, a_{n}$. For convenience, choose $\gamma_{i}$ 's such that $h\left(s_{i}^{j}\right)-h\left(t_{i}^{j}\right)=-1$ for $i$ odd, and $h\left(s_{i}^{j}\right)-h\left(t_{i}^{j}\right)=1$ for $i$ even. Thus we have the following system of linear equations:

$$
\begin{gathered}
\left(\left(s_{1}^{i}\right)^{N+1}-\left(t_{1}^{i}\right)^{N+1}\right) \gamma_{1}+\left(\left(s_{1}^{i}\right)^{N}-\left(t_{1}^{i}\right)^{N}\right) \gamma_{2}+\cdots+\left(\left(s_{1}^{i}\right)-\left(t_{1}^{i}\right)\right) \gamma_{N+1} \\
=\left(t_{1}^{i}\right)^{N+2}-\left(s_{1}^{i}\right)^{N+2}-1, \\
\left(\left(s_{2}^{i}\right)^{N+1}-\left(t_{2}^{i}\right)^{N+1}\right) \gamma_{1}+\left(\left(s_{2}^{i}\right)^{N}-\left(t_{2}^{i}\right)^{N}\right) \gamma_{2}+\cdots+\left(\left(s_{2}^{i}\right)-\left(t_{2}^{i}\right)\right) \gamma_{N+1} \\
\vdots \\
\left.\left(\left(s_{a_{i}}^{i}\right)^{N+1}-\left(t_{a_{i}}^{i}\right)^{N+1}\right) \gamma_{1}+\ldots+\left(\left(s_{a_{i}}^{i}\right)-\left(t_{a_{i}}^{i}\right)\right) \gamma_{N+1}\right)^{N+2}-\left(s_{2}^{i}\right)^{N+2}+1, \\
=\left(t_{a_{i}}^{i}\right)^{N+2}-\left(s_{a_{i}}^{i}\right)^{N+2}+(-1)^{a_{i}},
\end{gathered}
$$

for $i=1, \ldots, n$. This is a system of $\sum_{i=1}^{n} a_{i}=N$ linear equations in $N+1$ variables. Its coefficient matrix is

$$
B=\left(\begin{array}{cccc}
\left(s_{1}^{1}\right)^{N+1}-\left(t_{1}^{1}\right)^{N+1} & \left(s_{1}^{1}\right)^{N}-\left(t_{1}^{1}\right)^{N} & \ldots & s_{1}^{1}-t_{1}^{1} \\
\left(s_{2}^{1}\right)^{N+1}-\left(t_{2}^{1}\right)^{N+1} & \left(s_{2}^{1}\right)^{N}-\left(t_{2}^{1}\right)^{N} & \ldots & s_{2}^{1}-t_{2}^{1} \\
\vdots & \vdots & \vdots & \vdots \\
\left(s_{a_{1}}^{1}\right)^{N+1}-\left(t_{a_{1}}^{1}\right)^{N+1} & \left(s_{a_{1}}^{1}\right)^{N}-\left(t_{a_{1}}^{1}\right)^{N} & \ldots & s_{a_{1}}^{1}-t_{a_{1}}^{1} \\
\left(s_{1}^{2}\right)^{N+1}-\left(t_{1}^{2}\right)^{N+1} & \left(s_{1}^{2}\right)^{N}-\left(t_{1}^{2}\right)^{N} & \ldots & s_{1}^{2}-t_{1}^{2} \\
\vdots & \vdots & \vdots & \vdots \\
\left(s_{a_{2}}^{2}\right)^{N+1}-\left(t_{a_{2}}^{2}\right)^{N+1} & \left(s_{a_{2}}^{2}\right)^{N}-\left(t_{a_{2}}^{2}\right)^{N} & \ldots & s_{a_{2}}^{2}-t_{a_{2}}^{2} \\
\vdots & \vdots & \vdots & \vdots \\
\left(s_{a_{n}}^{n}\right)^{N+1}-\left(t_{a_{n}}^{n}\right)^{N+1} & \left(s_{a_{n}}^{n}\right)^{N}-\left(t_{a_{n}}^{n}\right)^{N} & \ldots & s_{a_{n}}^{n}-t_{a_{n}}^{n}
\end{array}\right) .
$$

The above system will have a solution if we can ensure that the rank of $B$ is $N$. But $A$ is clearly a non-singular submatrix of $B$. Hence the above system always has a solution. In fact, we can also find a polynomial $h(t)$ of degree more than $N+2$, which still provides an over/under crossing data for the corresponding 2-bridge knot. Thus $(f(t), g(t), h(t))$ is a polynomial representation for a 2-bridge knot with $N$ crossings.

To justify the minimality of this degree sequence we proceed as follows. We first notice that the degree of $f(t)$ cannot be made less than 3 since otherwise the knot represented by $t \mapsto(f(t), g(t), h(t))$ is equivalent to the 
trivial knot. Also from Lemma 2.4 of [7] the degree of $h(t)$ should be at least $N+1$. Now, we divide the argument into cases:

CASE 1: $N \equiv 0(\bmod 3)$. Here $N+1$ and $N+2$ are co-prime to 3 and the triple $(3, N+1, N+2)$ satisfies the condition that none of the coordinates lie in the semigroup generated by the other two. Also, if we reduce any of the degrees, by a polynomial automorphism it will cut down to a degree sequence which cannot represent the desired 2-bridge knot. Thus $(3, N+1, N+2)$ is the minimal degree sequence in this case.

CASE $2: N \equiv 1(\bmod 3)$. In this case $N+1$ is co-prime to 3 , but $N+$ 2 is divisible by 3 . Thus if we take a polynomial embedding with degree $(3, N+1, N+2)$ then by a polynomial automorphism we will be able to cut down these degrees to $(3, N, N+1)$, which cannot represent a 2-bridge knot with $N$ crossings. Therefore, we take the polynomial $h(t)$ to be of degree $N+3$. Using the theory of linear equations, and the above argument, we can find a polynomial $h(t)$ of degree $N+3$ which provides the over/under crossing data for this knot. As in the previous case, we can show that $(3, N+1, N+3)$ is the minimal degree sequence.

CASE $3: N \equiv 2(\bmod 3)$. In this case $N+1$ is divisible by 3 , but $N+2$ is not. Here we choose

$$
g(t)=t^{N+3}+\beta_{1} t^{N+2}+\cdots+\beta_{N+3} .
$$

Choose $s_{j}^{i}$ for $i=1, \ldots, n$ and $j=1, \ldots, a_{i}$, and the corresponding $t_{j}^{i}$ such that $f\left(s_{j}^{i}\right)=f\left(t_{j}^{i}\right)$. Also choose a complex number $s_{0}+i t_{0}$ and the corresponding $s_{0}^{1}+i t_{0}^{1}$ such that $f\left(s_{0}+i t_{0}\right)=f\left(s_{0}^{1}+i t_{0}^{1}\right)$, hence $f\left(s_{0}-i t_{0}\right)=$ $f\left(s_{0}^{1}-i t_{0}^{1}\right)$. Now choose the coefficients $\beta_{1}, \ldots, \beta_{N+3}$ of $g(t)$ such that

$$
\begin{aligned}
& g\left(s_{j}^{i}\right)=g\left(t_{j}^{i}\right) \quad \text { for } i=1, \ldots, n \text { and } j=1, \ldots, a_{i}, \\
& g\left(s_{0}+i t_{0}\right)=g\left(s_{0}^{1}+i t_{0}^{1}\right) .
\end{aligned}
$$

The last equation gives two linear equations by equating the real and imaginary parts. Hence we get a system of $N+2$ equations in $N+2$ variables. By a similar argument as before we can ensure the existence of a solution for $\beta_{1}, \ldots, \beta_{N+3}$.

Thus we can get a regular projection of this knot by $t \mapsto(f(t), g(t))$ where $\operatorname{deg} f(t)=3$ and $\operatorname{deg} g(t)=N+3$. Let $\left(s_{j}^{i}, t_{j}^{i}\right)$ be points of intersections.

Let $h(t)=t^{N+2}+\gamma_{1} t^{N+1}+\cdots+\gamma_{N+2}$. Choose $\gamma_{i}$ such that

$$
\begin{array}{ll}
h\left(s_{i}^{j}\right)-h\left(t_{i}^{j}\right)=-1 & \text { for } i \text { odd, } \\
h\left(s_{i}^{j}\right)-h\left(t_{i}^{j}\right)=+1 & \text { for } i \text { even. }
\end{array}
$$

This gives a system of $N$ equations in $N+1$ variables and a choice of $s_{j}^{i}$ can be made such that the rank of the coefficient matrix for the system (I) and 
the system (II) can be simultaneously made to be $N+2$ and $N$ respectively. Thus we have $h(t)$ of degree $N+2$ and $(3, N+2, N+3)$ is a degree sequence for this knot. Note that this regular projection is into the $X Z$-plane. This degree sequence is minimal because if we reduce any of these degrees, by earlier arguments we can show that it will not be possible to represent this knot.

4. Examples. In this section we apply our theorem to some examples and demonstrate how to obtain a polynomial representation of these 2-bridge knots with minimal degree sequence.

EXAMPLE 4.1 (A 2-bridge knot with four crossings). This is indeed our figure eight knot. When we represent it as a rational knot in Conway's normal form we see that a 2-bridge knot with four crossings must be either $C(2,1,1)$ or $C(1,1,2)$ and we can easily check that they are equivalent. By our main theorem the minimal degree sequence of this knot must be $(3,5,7)$.

In order to obtain a polynomial representation of this knot with the degree sequence $(3,5,7)$ we consider a regular diagram for this knot as shown in Figure 12 and its projection into the $X Y$-plane as shown in Figure 13.

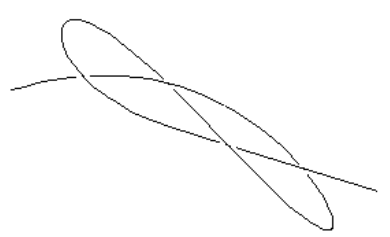

Fig. 12

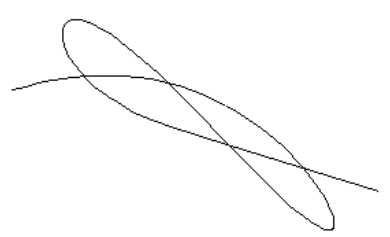

Fig. 13

Take $X(t)=f(t)=t\left(t^{2}-16\right)$. Choose

$$
s_{1}=-4.53723, \quad s_{2}=-4.16121, \quad s_{3}=-3.82946, \quad s_{4}=0.010048
$$

and find the corresponding

$$
t_{1}=3.01704, \quad t_{2}=0.344731, \quad t_{3}=4.15112, \quad t_{4}=3.99497
$$

such that $f\left(s_{i}\right)=f\left(t_{i}\right)$ for $i=1,2,3,4$. Let

$$
g(t)=t^{5}+\alpha_{1} t^{4}+\alpha_{2} t^{3}+\alpha_{3} t^{2}+\alpha_{4} t .
$$

We have to determine $\alpha_{1}, \alpha_{2}, \alpha_{3}, \alpha_{4}$ such that $g\left(s_{i}\right)=g\left(t_{i}\right)$ for $i=1,2,3,4$. This gives us the following system of linear equations:

$$
\begin{aligned}
& \left(s_{1}^{4}-t_{1}^{4}\right) \alpha_{1}+\left(s_{1}^{3}-t_{1}^{3}\right) \alpha_{2}+\left(s_{1}^{2}-t_{1}^{2}\right) \alpha_{3}+\left(s_{1}-t_{1}\right) \alpha_{4}=t_{1}^{5}-s_{1}^{5}, \\
& \left(s_{2}^{4}-t_{2}^{4}\right) \alpha_{1}+\left(s_{2}^{3}-t_{2}^{3}\right) \alpha_{2}+\left(s_{2}^{2}-t_{2}^{2}\right) \alpha_{3}+\left(s_{2}-t_{2}\right) \alpha_{4}=t_{2}^{5}-s_{2}^{5}, \\
& \left(s_{3}^{4}-t_{3}^{4}\right) \alpha_{1}+\left(s_{3}^{3}-t_{3}^{3}\right) \alpha_{2}+\left(s_{3}^{2}-t_{3}^{2}\right) \alpha_{3}+\left(s_{3}-t_{3}\right) \alpha_{4}=t_{3}^{5}-s_{3}^{5}, \\
& \left(s_{4}^{4}-t_{4}^{4}\right) \alpha_{1}+\left(s_{4}^{3}-t_{4}^{3}\right) \alpha_{2}+\left(s_{4}^{2}-t_{4}^{2}\right) \alpha_{3}+\left(s_{4}-t_{4}\right) \alpha_{4}=t_{4}^{5}-s_{4}^{5} .
\end{aligned}
$$


This system has a unique solution if the rank of the coefficient matrix is 4 , i.e. if $\operatorname{det}(M) \neq 0$. Substituting the above values of $s_{i}$ and $t_{i}$ we find that

$$
M=\left(\begin{array}{cccc}
340.946 & -120.868 & 11.4839 & -7.55427 \\
299.818 & -72.0951 & 17.1968 & -4.50594 \\
-81.8795 & -127.689 & -2.56703 & -7.98058 \\
-254.715 & -63.7589 & -15.9597 & -3.98492
\end{array}\right)
$$

and $\operatorname{det}(M)=3.07914$. Hence the above system has a unique solution. After obtaining the solution of this system we find that $g(t)$ is given by

$$
g(t)=t^{5}+1.01 t^{4}-33.8144 t^{3}-14.0925 t^{2}+277.556 t .
$$

The parametric plot of $(f(t), g(t))$ (drawn using Mathematica) is shown in Figure 14.

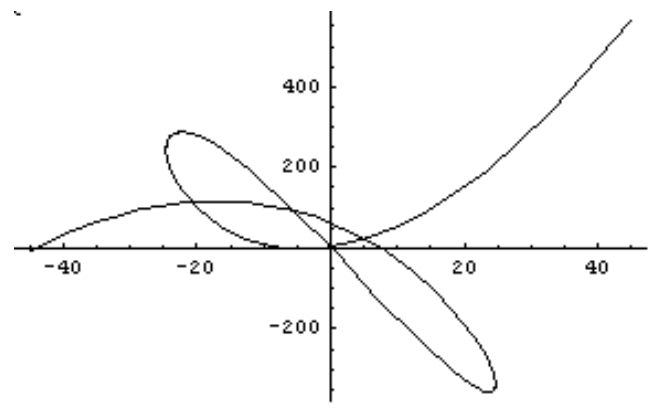

Fig. 14

Now, we want to construct a polynomial $h(t)$ which provides an over/under crossing data for this 2-bridge knot. Let

$$
h(t)=t^{7}+\beta_{1} t^{6}+\beta_{2} t^{5}+\beta_{3} t^{4}+\beta_{4} t^{3}+\beta_{5} t^{2}+\beta_{6} t .
$$

Since our knot is alternating, we want

$$
h\left(s_{i}\right)-h\left(t_{i}\right)<0 \quad \text { for } i \text { odd, } \quad h\left(s_{i}\right)-h\left(t_{i}\right)>0 \quad \text { for } i \text { even. }
$$

For convenience, take $h\left(s_{i}\right)-h\left(t_{i}\right)=-1$ for $i$ odd, and $h\left(s_{i}\right)-h\left(t_{i}\right)=1$ for $i$ even. Thus we obtain four linear equations in six variables. By solving these equations we get $\beta_{1}=-13285.2, \beta_{2}=-241602.1, \beta_{3}=181162$, $\beta_{4}=3.97824 \cdot 10^{6}, \beta_{5}=2986.5841178525206$ and $\beta_{6}=12.084074534654706$. Hence

$$
\begin{aligned}
h(t)= & t^{7}-13285.2 t^{6}-241602 t^{5}+181162 t^{4}+3.97824 \cdot 10^{6} t^{3} \\
& +2986.584 t^{2}+12.084075 t
\end{aligned}
$$

Thus $(f(t), g(t), h(t))$ is a polynomial representation for the 2-bridge knot $[2,1,1]$. A 3D plot of this knot is shown in Figure 15. 


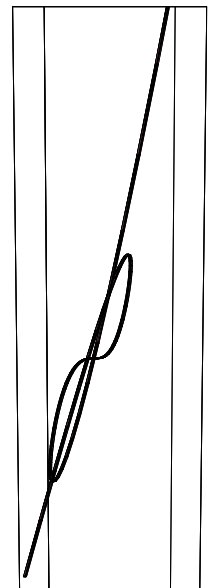

Fig. 15

ExAmPle 4.2 (A 2-bridge knot with five crossings). In Conway's normal form it is $C(2,2,1)$ and by our theorem its minimal degree sequence is $(3,7,8)$.

A regular diagram of this knot and its projection are shown in Figure 16 and Figure 17 respectively.

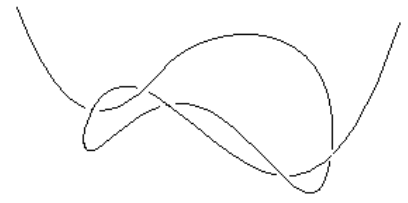

Fig. 16

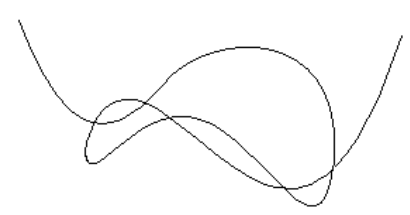

Fig. 17

Let $f(t)=t\left(t^{2}-16\right)$. We choose $s_{1}=-4.36684, s_{2}=-4.29429, s_{3}=$ $-2.74018, s_{4}=-0.957032$ and $s_{5}=0.413846$ and the corresponding $t_{1}=$ $0.880321, t_{2}=3.62001, t_{3}=4.89012, t_{4}=4.39171$, and $t_{5}=3.77699 \mathrm{such}$ that $f\left(s_{i}\right)=f\left(t_{i}\right)$ for $i=1, \ldots, 5$. Let

$$
g(t)=t^{8}+\alpha_{1} t^{7}+\alpha_{2} t^{6}+\cdots+\alpha_{6} t^{2}+\alpha_{7} t,
$$

where the coefficients $\alpha_{i}$ of $g(t)$ are to be determined. Since a polynomially parametrized plane curve $(X(t), Y(t))=(f(t), g(t))$ can have seven nodes whereas in the projection of this knot we require only five, we choose a complex number $s_{0}+i s_{0}^{\prime}=-5.00845-i 0.784492$ and the corresponding $t_{0}+i t_{0}^{\prime}=1.09112+i 2.4776$ such that $f\left(s_{0}+i s_{0}^{\prime}\right)=f\left(t_{0}+i t_{0}^{\prime}\right)$ and hence $f\left(s_{0}-i t_{0}\right)=f\left(s_{0}^{\prime}-i t_{0}^{\prime}\right)$. Now we impose the condition that $g\left(s_{i}\right)=g\left(t_{i}\right)$ for each $i=1, \ldots, 5$ and $g\left(s_{0}+i s_{0}^{\prime}\right)=g\left(t_{0}+i t_{0}^{\prime}\right)$. By solving the linear 
equations

$$
g\left(s_{0}\right)=g\left(t_{0}\right), \quad g\left(s_{0}^{\prime}\right)=g\left(t_{0}^{\prime}\right), \quad g\left(s_{i}\right)=g\left(t_{i}\right) \quad \text { for } i=1,2,3,4,5,
$$

we will get

$$
\begin{aligned}
g(t)= & 8-0.497173 t^{7}-46.9362 t^{6}+34.4793 t^{5}+649.263 t^{4} \\
& -544.114 t^{3}-2327.38 t^{2}+1120.43 t
\end{aligned}
$$

The parametric plot of $(f(t), g(t))$ (drawn using Mathematica) is shown in Figure 18.

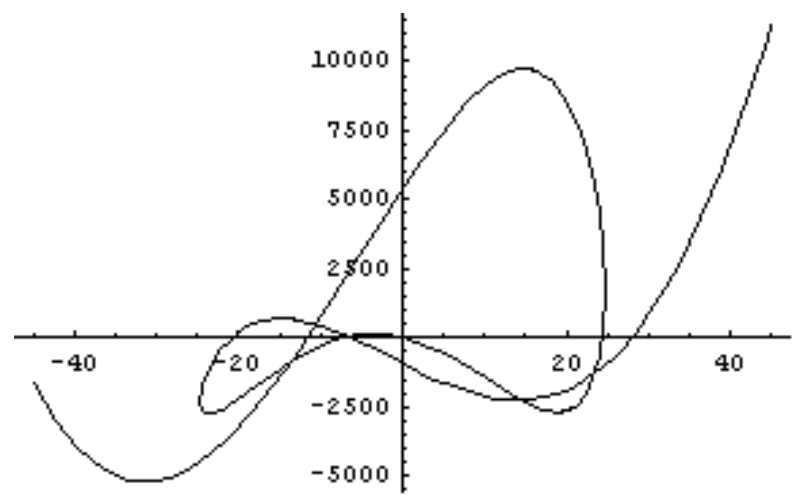

Fig. 18

Again by a similar method we find the polynomial

$$
\begin{aligned}
h(t)= & t^{7}-97.8602 t^{6}-854.142 t^{5}+5766.69 t^{4}+7931.55 t^{3} \\
& -77465.7 t^{2}+125398.88 t .
\end{aligned}
$$

A 3D picture of this knot is shown in Figure 19.

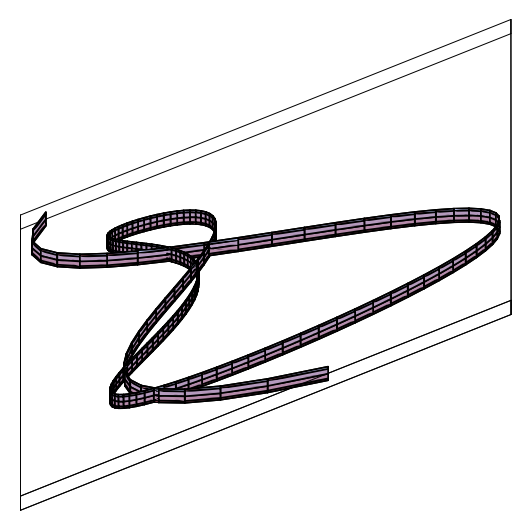

Fig. 19 
EXAMPLE 4.3 (A 2-bridge knot with six crossings). Let us take the rational knot in Conway's normal form to be $C(2,1,1,1,1)$. A regular diagram of this knot and its projection are shown in Figures 20 and 21 respectively.

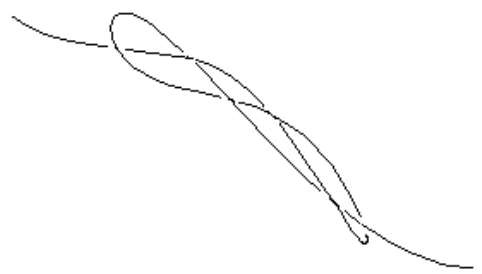

Fig. 20

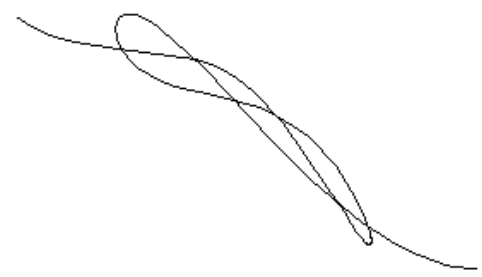

Fig. 21

Let $X(t)=f(t)=t\left(t^{2}-16\right)$. We choose the points $s_{1}=-4.50895$, $s_{2}=-4.26245, s_{3}=-3.78869, s_{4}=-3.23304, s_{5}=-1.99952$ and $s_{6}=$ 0.0984486 and obtain the corresponding $t_{1}=1.38728, t_{2}=3.67189, t_{3}=$ $-0.39353, t_{4}=4.47319, t_{5}=4.60551$ and $t_{6}=3.94987$ such that $f\left(s_{i}\right)=$ $f\left(t_{i}\right)$ for all $i$.

Let $g(t)=t^{7}+\alpha_{1} t^{6}+\alpha_{2} t^{5}+\alpha_{3} t^{4}+\alpha_{4} t^{3}+\alpha_{5} t^{2}+\alpha_{6} t+\alpha_{7}$. We want to choose the coefficients of $g(t)$ such that $g\left(s_{i}\right)=g\left(t_{i}\right)$ for all $i$.

Using the theory of linear equations we obtain $\alpha_{1}=-2.32015, \alpha_{2}=$ $-37.8493, \alpha_{3}=68.303, \alpha_{4}=294.038, \alpha_{5}=-486.111$, and $\alpha_{6}=787.942$. Hence

$g(t)=t^{7}-2.32015 t^{6}-37.8493 t^{5}+68.303 t^{4}+294.038 t^{3}-486.111 t^{2}+787.942 t$. The parametric plot of $(f(t), g(t))$ (drawn using Mathematica) is shown in Figure 22.

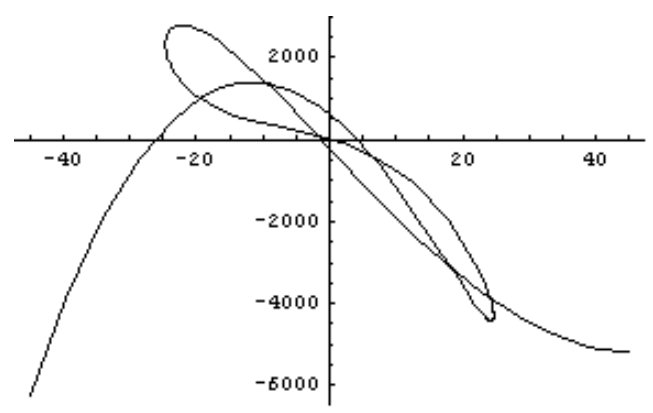

Fig. 22

Let $h(t)=t^{8}+\beta_{1} t^{7}+\cdots+\beta_{7} t+\beta_{8}$. We want to choose the coefficients $\beta_{i}$ of $h(t)$ such that $h\left(s_{i}\right)-h\left(t_{i}\right)<0$ for $i$ odd, and $h\left(s_{i}\right)-h\left(t_{i}\right)>0$ for $i$ even. Using the method as in earlier examples we obtain $\beta_{1}=1.51699$, $\beta_{2}=-57.9838, \beta_{3}=-54.5407, \beta_{4}=1045.86, \beta_{5}=821.517, \beta_{6}=-6025.3$, $\beta_{7}=-5353.43$ and $\beta_{8}=0$. 
We can observe that $(f(t), g(t), h(t))$ represents a 2-bridge knot of type $[2,1,1,1,1]$ and the degree sequence $(3,7,8)$ is the minimal degree sequence. A 3D plot of this knot is shown in Figure 23.

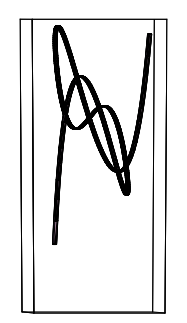

Fig. 23

Acknowledgements. We would like to thank Prof. Józef Przytycki for suggesting this problem. Thanks are also due to Prof. A. Ranjan for reading the manuscript and offering valuable suggestions.

\section{References}

[1] G. Burde and H. Zieschang, Knots, de Gruyter Stud. Math. 5, de Guyter, 1985.

[2] L. H. Kauffman and S. Lambropoulou, On the classification of rational tangles, Adv. Appl. Math. 33 (2004), 199-237.

[3] - - - On the classification of rational knots, Enseign. Math. 49 (2003), 357-410.

[4] K. Murasugi, Knot Theory and its Applications, translated from the 1993 Japanese original by B. Kurpita, Birkhäuser, 1996.

[5] P. Madeti and R. Mishra, Minimal degree sequence for torus knots of type $(p, 2 p-1)$, J. Knot Theory Ramif., to appear; http://www.worldscinet.com/jktr/editorial/paper/750349.pdf.

[6] R. Mishra, Polynomial representation of torus knots of type $(p, q)$, J. Knot Theory Ramif. 8 (1999), 667-700.

[7] —, Minimal degree sequence for torus knots, ibid. 9 (2000), 759-769.

[8] A. Ranjan and R. Shukla, On polynomial representation of torus knots, ibid. 5 (1996), 279-294.

[9] D. Rolfsen, Knots and Links, Publish or Perish, Berkeley, 1976.

[10] A. R. Shastri, Polynomial representations of knots, Tohoku Math. J. 44 (1992), 11-17.

Department of Mathematics

Indian Institute of Technology Delhi

Hauz Khas, New Delhi 110 016, India

E-mail: mprabhakar_iitd@yahoo.co.in rama@maths.iitd.ac.in 